\title{
A Novel based Cooperation and Joint Source- Channel Transmission in Wireless Networks using LDPC
}

\author{
Areplli Siva Parvathi \\ Student, \\ Pursuing masters in \\ engineering in DECS \\ Gudlavalleru college of \\ Engineering / JNTU \\ Kakinada University, AP, India
}

\author{
G. Rama Krishna \\ Assistant Professor, \\ Gudlavalleru college of \\ Engineering / JNTU \\ Kakinada University, AP, India
}

\author{
M. Kamaraju, Ph.D. \\ Professor, \\ Gudlavalleru college of \\ Engineering / JNTU \\ Kakinada University, AP, India
}

\begin{abstract}
In this paper, A Novel based Cooperation and joint source channel Transmission in wireless networks in this paper Low density parity check (LDPC) codes is linear different number of the forward error correcting codes, method of transmitting the number of messages over noisy transmission of a channel . Low density parity check (LDPC) codes are mainly used to minimize the outage probability. We summarize the methods based on the Read Solomon (RS) codes and the Progressive Edge Growth (PEG) algorithm in selection. The firest,we select a profile that describes that desire the desired number of columns of each weight and the desired number of rows of each weight. The symbols produced by the information source are given to the source encoder. These symbols cannot be transmitted directly. They are first concerted into digital form .The source encoder assign codeword's to the symbols for each discrete symbol there is an unique codeword. The transmission errors basically are of two types namely the random errors and burst errors. The errors that occurs in a purely random manner are known as random errprs. Where as the errors which occur in the form of bunches and hence which the not independent are known as the burst errors. Codes like binary $\mathrm{BCH}$ codes are useful in dealing with the random eror.Because of impulse noise, more than one corresponding data bits change their state simultaneously. The error introduced in the received word is called as burst error. The channel encoder and decoder is after converting the message (or) information signal in the form of binary sequence by source encoder. The signal is to transmit through channel. The communication channel adds some noise and interference to the signal being transmitted. Hence errors are introduced in the binary sequence received at the receiver end.
\end{abstract}

\section{Index Terms}

Delay limited capacity, Outage capacity, power control fading channel, Quasi stationary sources, outage distortion of the channel, source coding and channel coding, rate of the channel and power adaptation, Low density parity check codes (LDPC).

\section{INTRODUCTION}

Low Density Parity Check (LDPC) codes are mainly used to the forward error correction codes. As the name suggest, the LDPC codes are block codes defined as parity check matrix that is sparge.They are first time proposed in the year of 1962 by the great scientist Gallager.

The Gallager is to proposed in his Ph.D thesis in the year of 1962 at Massachusetts Institute of Technology (MIT). At that time of their increasing the potential of the LDPC codes to performance and simulation in the vacuum tubes, these tubes is to transmit the digital signal. In 1995they were rediscovered by MacKay and Neal sparking a flurry of farther research.
The symbols produced by the information source are given to the source encoder. These symbols cannot be transmitted directly. They are first concerted into digital form .The source encoder assign codeword's to the symbols for each discrete symbol there is an unique codeword.

The channel encoder and decoder is after converting the message (or) information signal in the form of binary sequence by source encoder. The signal is to transmit through channel. The communication channel adds some noise and interference to the signal being transmitted. Hence errors are introduced in the binary sequence received at the receiver end.

The transmission errors basically are of two types namely the random errors and burst errors. The errors that occurs in a purely random manner are known as random errprs. Where as the errors which occur in the form of bunches and hence which the not independent are known as the burst errors. Codes like binary $\mathrm{BCH}$ codes are useful in dealing with the random eror.Because of impulse noise, more than one corresponding data bits change their state simultaneously. The error introduced in the received word is called as burst error.

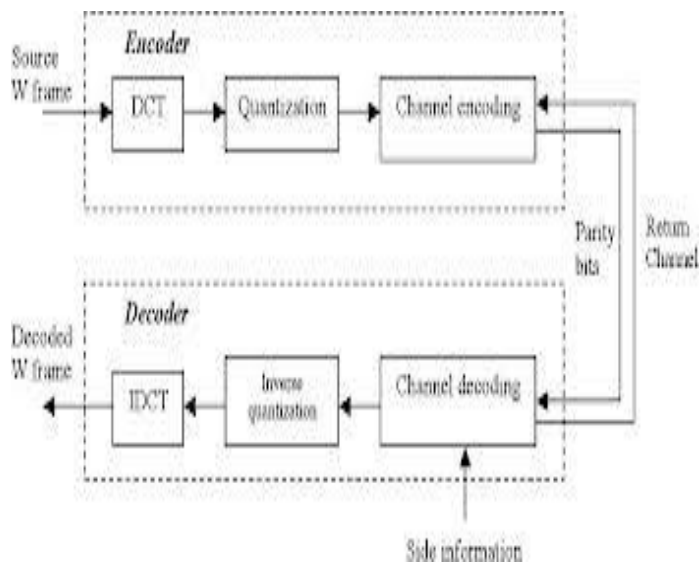

Fig 1 Low density parity check (LDPC) codes

\subsection{Source Channel Coding Techniques}

The source coding theorem states that for a discrete memory less source $X$, with entropy of the symbol $H(X)$, the average code word length $\mathrm{L}$ per symbol is bounded as

$$
\mathrm{L}>=\mathrm{H}(\mathrm{X})
$$

Low density parity check (LDPC) codes are mainly used to the forward error correction codes. Low density parity check (LDPC) codes are used to minimize outage probability.

The Shannon Hartley low underscores the fundamental rate of bandwidth and signal to noise $\operatorname{ratio}(\mathrm{S} / \mathrm{N})$ in communication. It is also shows that we can exchange increased bandwidth for 
decreased signal power for a system with given capacity $\mathrm{C}$. The low density parity check (LDPC) codes were discovered by the great scientist Gallager in the year of 1960's.

Coding is the most important application of information theory. When digital data form an information source is transmitted, it experiences various types of noise, distortions and interference in the channel before reaching the destination. Due to this the received data is erroneously received. Hence, there is a need for error control, which involves error detecting and then error correction.

To achieve this coding of the information signal is required. The main purpose of coding is to improve the efficiency of the digital communication system, in the sense, improve the bit error rate (BER) at the output of the receiver.

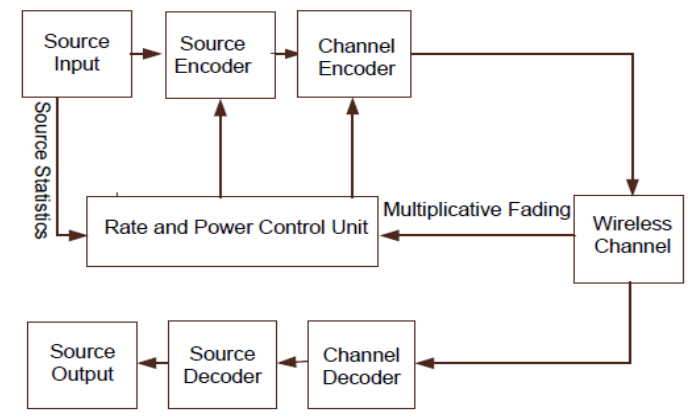

Fig 2 Block diagram of the system

The symbols produced by the information source are given to the source encoder. These symbols cannot be transmitted directly. They are first concerted into digital form .The source encoder assign codeword's to the symbols for each discrete symbol there is an unique codeword.

The channel encoder and decoder is after converting the message (or) information signal in the form of binary sequence by source encoder. The signal is to transmit through channel. The communication channel adds some noise and interference to the signal being transmitted. Hence errors are introduced in the binary sequence received at the receiver end

The transmission errors, basically are of two types namely the random errors and burst errors. The errors that occurs in a purely random manner are known as random errprs. Where as the errors which occur in the form of bunches and hence which the not independent are known as the burst errors. Codes like binary $\mathrm{BCH}$ codes are useful in dealing with the random eror.Because of impulse noise, more than one corresponding data bits change their state simultaneously. The error introduced in the received word is called as burst error.

\subsection{LDPC Encoding}

In LDPC codes noted that the Generating matrix [G] and Parity check matrix is $[\mathrm{H}]$.The two matrix can be used by performance of gauss Jordon elimination on the Parity check Matrix $[\mathrm{H}]$ is in the form of

$$
H=\left|A_{1} I_{n-k}\right|
$$

The above equation A is binary matrix of order of (n-1) and identity matrix I of the order of (n-k).The Generator matrix G is represented in the form
The above equation A is binary matrix of order of $\mathrm{k}$ and $\mathrm{I}$ is identity matrix of order k.In LDPC codes encoded the length of rate to rate $-1 / 2$.

$$
\begin{aligned}
& \mathrm{H}=\left[\begin{array}{llllllllll}
1 & 1 & 0 & 1 & 1 & 0 & 0 & 1 & 0 & 0
\end{array}\right. \\
& 0110111000 \\
& 0001000111 \\
& 1100011010 \\
& 0001000100101] \text {. }
\end{aligned}
$$

First, the above parity check matrix $[\mathrm{H}]$ put into row echelon form (i.e. so that in any two successive rows of the Parity matrix $\mathrm{H}$ that do not consists of entirely of zeros, the leading 1 's two sum of the 5th and 4th rows gives $\mathrm{Hr}$ in row

$$
H_{r}=\left[\begin{array}{llllllllll}
1 & 1 & 0 & 1 & 1 & 0 & 0 & 1 & 0 & 0 \\
0 & 1 & 1 & 0 & 1 & 1 & 1 & 0 & 0 & 0 \\
0 & 0 & 1 & 0 & 0 & 1 & 0 & 1 & 0 & 1 \\
0 & 0 & 0 & 1 & 1 & 1 & 1 & 1 & 1 & 0 \\
0 & 0 & 0 & 0 & 1 & 1 & 1 & 0 & 0 & 1
\end{array}\right] .
$$

The next parity check matrix $[\mathrm{H}]$.In the parity check matrix is put in the rows echelon form. The $1^{\text {st }}$ column of the parity check matrix is already corrected and entry in to the $2^{\text {nd }}$ column of the parity check matrix the above diagonal is to be remove in the 2 nd column and replacing the $1^{\text {st }}$ row in the above matrix.

$$
H_{\pi}=\left[\begin{array}{llllllllll}
1 & 0 & 0 & 0 & 0 & 0 & 1 & 1 & 1 & 0 \\
0 & 1 & 0 & 0 & 0 & 1 & 0 & 1 & 0 & 0 \\
0 & 0 & 1 & 0 & 0 & 1 & 0 & 1 & 0 & 1 \\
0 & 0 & 0 & 1 & 0 & 0 & 0 & 1 & 1 & 1 \\
0 & 0 & 0 & 0 & 1 & 1 & 1 & 0 & 0 & 1
\end{array}\right]
$$

The above parity check matrix in the form of Hstd,

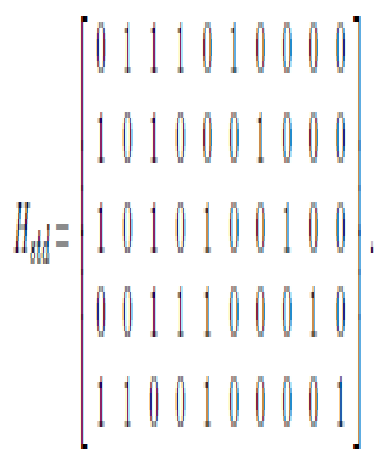

In the final stage column permutations have been used and so the codeword of the matrix Hstd will be the same code words corresponding in the parity check matrix. The problem is solved is keep tracking the column and create the matrix Hstd.the matrix Hstd is written as the

$$
\Pi=\left[\begin{array}{llllllllll}
6 & 7 & 8 & 9 & 10 & 1 & 2 & 3 & 4 & 5
\end{array}\right]
$$

$$
G=\left[I_{k}, A^{T}\right] .
$$


And apply the inverse of the parity check $[\mathrm{H}]$ matrix. The matrix Hstd and code word before it is transmitted codeword's (0's and 1's). The channel is memory less channel, and so the order of code word (0's and 1's) bits un important, a for easier option is to apply to original parity check matrix $[\mathrm{H}]$ to give parity check matrix

$$
H^{\prime}=\left[\begin{array}{llllllllll}
1 & 1 & 0 & 1 & 1 & 0 & 0 & 1 & 0 & 0 \\
0 & 1 & 1 & 0 & 1 & 1 & 1 & 0 & 0 & 0 \\
0 & 0 & 0 & 1 & 0 & 0 & 0 & 1 & 1 & 1 \\
1 & 1 & 0 & 0 & 0 & 1 & 1 & 0 & 1 & 0 \\
0 & 0 & 1 & 0 & 0 & 1 & 0 & 1 & 0 & 1
\end{array}\right]
$$

With the same properties as the parity check matrix $[\mathrm{H}]$ but which the transmitted same code word bits ordering as the matrix Hstd. Finally generate matrix $G$ for the transmitted codeword's with parity check matrices is the Hstd and the parity check matrix H' is represented in the form of

$$
G=\left[\begin{array}{llllllllll}
1 & 0 & 0 & 0 & 0 & 0 & 1 & 1 & 0 & 1 \\
0 & 1 & 0 & 0 & 0 & 1 & 0 & 0 & 0 & 1 \\
0 & 0 & 1 & 0 & 0 & 1 & 1 & 1 & 1 & 0 \\
0 & 0 & 0 & 1 & 0 & 1 & 0 & 0 & 1 & 0 \\
0 & 0 & 0 & 0 & 1 & 0 & 0 & 1 & 1 & 1
\end{array}\right] .
$$

The above Generator matrix $G$ of the Processing in the form of off line process and the Generator matrix $G$ is encoding and Parity check matrix $\mathrm{H}$ is decoding. The main drawback of coding and decoding approach is that, Parity check matrix $\mathrm{H}$, the Generator matrix $\mathrm{G}$ will most

$$
H_{t}=\left[\begin{array}{lll}
A & B & T \\
O & D & E
\end{array}\right],
$$

The above matrix can be represented in $\mathrm{T}$ is lower triangular matrix

\section{ERROR CORRECTS TECHNIQUES}

The transmission errors basically are of two types namely the random errors and burst errors. The errors that occurs in a purely random manner are known as random errprs. Where as the errors which occur in the form of bunches and hence which the not independent are known as the burst errors. Codes like binary $\mathrm{BCH}$ codes are useful in dealing with the random eror.Because of impulse noise, more than one corresponding data bits change their state simultaneously. The error introduced in the received word is called as burst error

The transmission errors basically are of two types namely the random errors and burst errors. The errors that occurs in a purely random manner are known as random errprs. Where as the errors which occur in the form of bunches and hence which the not independent are known as the burst errors. Codes like binary $\mathrm{BCH}$ codes are useful in dealing with the random eror.Because of impulse noise, more than one corresponding data bits change their state simultaneously. The error introduced in the received word is called as burst error.

\subsection{Numerical Results by using LDPC Codes}

The below Fig 3 and 4 Depict Is DISTORTION OUTAGE PROBABILITY and Performance of the power constrant $(\mathrm{P})$ and out put power (Pout) for $\mathrm{Dm}=5 \mathrm{db}$ and $\mathrm{Dm}=2 \mathrm{db}$.

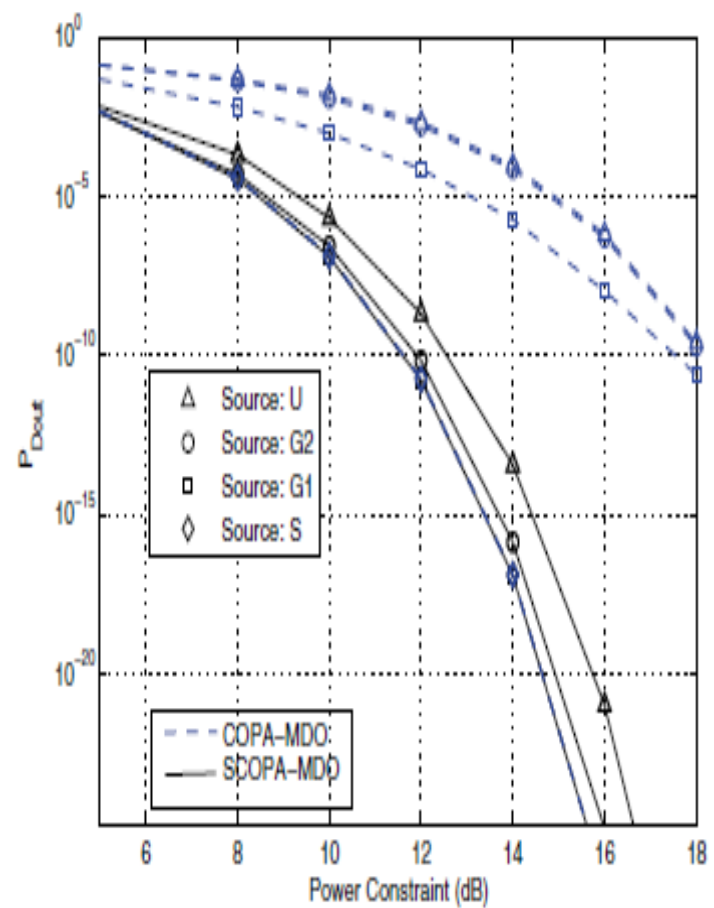

Fig.3 Distortion Outage probability vs power $(P)$ provided by the SCOPA at the MDO and COPA at the MDO for the five main different sources: $b=1$ and $D m=8 d B$.

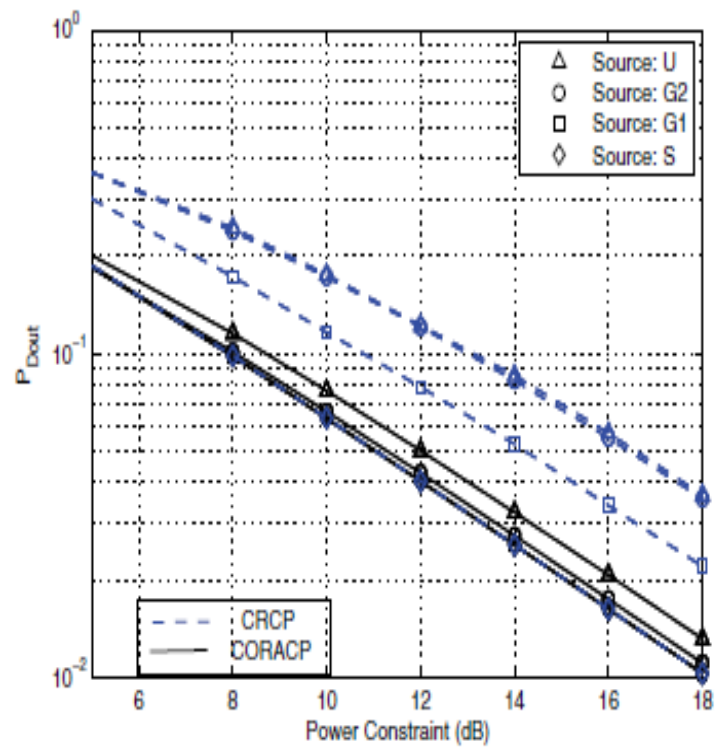

Fig. 4 is the distortion outage probability vs the power (P) and the provided by the CORACP nad the DRCP with for a five different sources: $b=1 \mathrm{db}$ and $\mathrm{Dm}=8 \mathrm{db}$. 


\subsection{Low Density Parity Check Codes (LDPC) Graphs}

outage probability versus Pbar provided by CORACP and CRCP for five different sources; $b=1$ ar

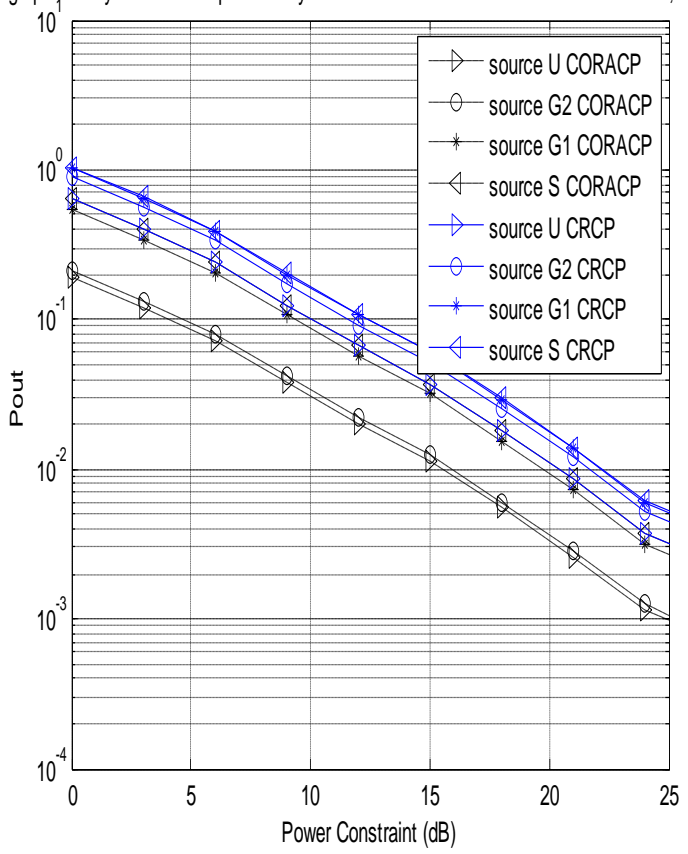

outage probability versus Pbar provided by CORACP and CRCP for five different sources; $b=1$ ar

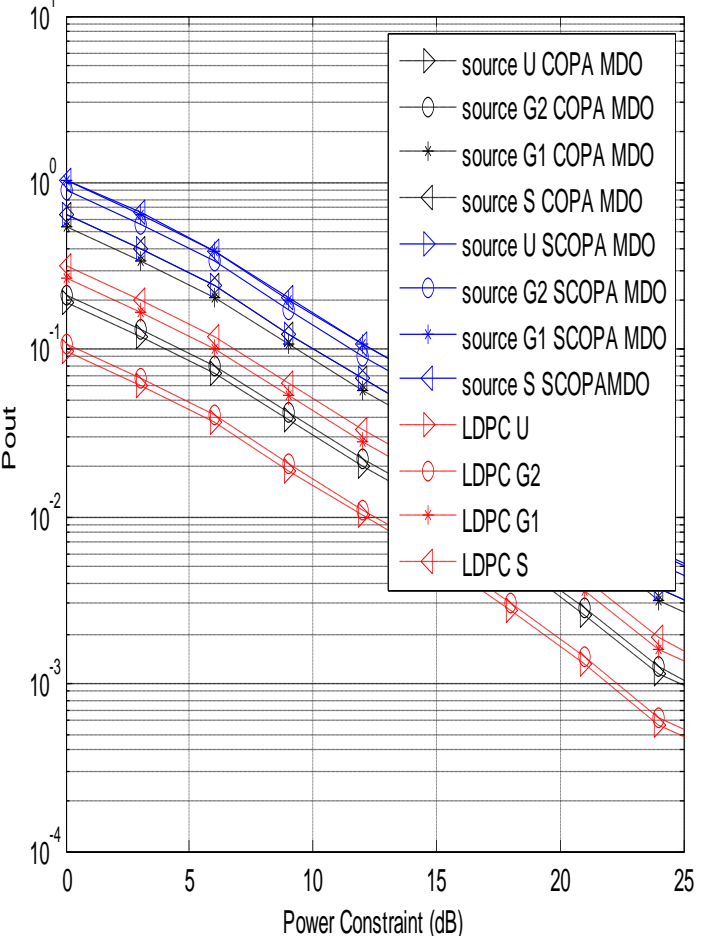

\section{CONCLUSION}

This paper is to introduce proposal and implementation for Low density parity checks (LDPC) codes are performance evaluation and design platform of LDPC codes. The Low density parity check (LDPC) codes are performance, simulation and design a platform of code words. This platform is to perform two main tasks. The first task is the performs parallel simulation and to the evaluate an Low density parity check (LDPC) codes performance.

The second task is, it graphical representations of the state of Low density parity check (LDPC) codes encoder and decoder. The encoder and decoder. The encoder and decoder are discussed with LDPC coding. The LDPC decoding may be best understand by the sum product algorithm and bit flipping algorithm showing the LDPC code can be implemented easily.

The simulation done for different rates are showing that are the short length LDPC codes are performing excellent, and the code perform better for all rates either low (or) high.

\section{REFERNCES}

[1] V.Hanly and D.Tse "part-I multi access fading channel. Part-II delay limited capacities ", IEEE Tran Inf Theory, vol .44, pp.2816-2831, Nov-1998.

[2] D. J. C. MacKay, "Good Error-Correcting Codes Based on very Sparse Matrices", IEEE Trans. Inform. Theory,45(1999), pp. 399-431.

[3] T. Richardson, "Error Floors of LDPC Codes", in Proc. 41st Annual Allerton Conf: On Communication, Computing and Control, Monticello, Oct. 2003.

[4] L. Li, N. Jindal, and A. Goldsmith, "Outage capacities and optimal power allocation for fading multiple-access channels," IEEE Trans. Inf.Theory, vol. 51, pp. 13261347, Nov. 2005

[5] T. Tian, C. Jones, J. D. Villasenor, and R. D. Wesel, "Construction of Irregular LDPC Codes With Low Error Floors", in Proc. IEEE International Conference on Communications, 5(2003), pp. 3125-3129.

[6] B. Dunn and J. N. Laneman, "Characterizing sourcechannel diversity approaches beyond the distortion 